\title{
Role of epidermal growth factor receptor in DNA damage repair
}

\author{
LU JingChen ${ }^{1,2,3}$, YANG LiFang ${ }^{1,3}$, TAO YongGuang ${ }^{1}$, SUN LunQuan ${ }^{3} \&$ CAO Ya ${ }^{1,3 *}$ \\ ${ }^{1}$ Key Laboratory of Carcinogenesis of Chinese Ministry of Public Health, Key Laboratory of Carcinogenesis and Cancer Invasion of Chinese \\ Ministry of Education, Cancer Research Institute, Xiangya School of Medicine, Central South University, Changsha 410078, China; \\ ${ }^{2}$ Department of Medical Oncology, Xiangya Hospital, Central South University, Changsha 410008, China; \\ ${ }^{3}$ Center for Molecular Medicine, Xiangya Hospital, Central South University, Changsha 410008, China
}

Received April 11, 2011; accepted June 21, 2011

\begin{abstract}
Many human tumor cells are characterized by overexpression or mutation of epidermal growth factor receptor (EGFR). Emerging evidence indicates that EGFR, as well as some of its downstream components, can translocate to the nucleus and play roles in transcriptional regulation, signaling conduction and repair of DNA double strands breaks (DSBs). EGFR in its nuclear manifestation promotes DSB repair by interacting with proteins including DNA-PK, ATM, Rad51 and BRCA1, involved in DSB repair, via the PI3K-Akt and Ras-Raf-MAPK pathways. DNA damage repair in tumor cells is emerging as an attractive target in radiotherapy and chemotherapy. Interruption of EGFR functions, or those of its downstream components, presents a promising strategy for confounding DNA damage repair in tumor cells.
\end{abstract}

epidermal growth factor receptor, nuclear translocation, ionizing radiation, DNA damage repair

Citation: $\quad$ Lu J C, Yang L F, Tao Y G, et al. Role of epidermal growth factor receptor in DNA damage repair. Chinese Sci Bull, 2011, 56: 3132-3137, doi: 10.1007/s11434-011-4650-5

The epidermal growth factor receptor (EGFR) belongs to the Type I subfamily of receptor tyrosine kinases (RTKs), which includes four closely related receptors: Her-1/ErbB1, Her-2/Neu/ErbB2, Her-3/ErbB3 and Her-4/ErbB4. EGFR is a $170-\mathrm{kD}$ transmembrane glycoprotein composed of 1186 aa residues. Structurally EGFR has an extracellular ligandbinding domain, a transmembrane domain, and an intracellular tyrosine kinase domain (TKD). The function and downstream signaling of EGFR as a cell surface receptor have been documented by a substantial body of literature. It is becoming abundantly clear, however, that aside from its duties in the cell membrane, EGFR and some of its downstream components translocate to the nucleus where they have various regulatory functions, such as transcriptional regulation and repair processes. Among these, the function of nuclear EGFR as a regulator of double strands breaks (DSBs) is of particular importance [1]. In this article, we review the current knowledge regarding EGFR modulation of DNA damage repair.

*Corresponding authors (email: ycao98@vip.sina.com; ljingchen@126.com)

\section{Activation and nuclear translocation of EGFR}

EGFR can be activated in ligand-dependent and ligandindependent manners. In response to genotoxic stresses, such as ionizing radiation (IR), cisplatin, UV light, heat shock and $\mathrm{H}_{2} \mathrm{O}_{2}$, EGFR is activated in the absence of a ligand and translocates to the nucleus [2,3]. Epstein-Barr virus (EBV)-encoded, latent membrane protein 1 (LMP1) has been shown to regulate the nuclear accumulation of EGFR in a ligand-independent manner, both quantitatively and qualitatively, in a nasopharyngeal carcinoma cell line, where it increased expression and phosphorylations of EGFR in a dose-dependent manner [4].

One effect of reactive oxygen species generated by ionizing radiation is the inactivation of a redox-sensitive, cysteine-based protein-tyrosine-phosphatase, which in turn causes a shift in the equilibrium between phosphorylated and dephosphorylated forms of EGFR and stimulates the kinase activity of the receptor in a ligand-independent 
manner. Another possible mechanism of EGFR activation in the irradiated cell is by up-regulation of autocrine/paracrine secretion of EGFR ligands [5].

Upon stimulation EGFR forms homodimers or heterodimers with some other ErbB family member, enabling its kinase activity and resulting in intermolecular phosphorylation of key tyrosine residues within the TKD and other proteins involved in downstream signaling. While both ligand-dependent and ligand-independent phosphorylations of EGFR trigger intracellular signaling and receptor internalization, the fate of receptor activated by these different modes is different. Indeed, binding of epidermal growth factor (EGF), the physiological ligand, results in the internalization of EGFR into clathrin-coated pits followed by receptor degradation while ionizing radiation results in a rapid stabilization and activation of Src kinase, and subsequent Src-mediated phoshorylation of Tyr14 of caveolin-1 and Tyr845 of EGFR. These phosphorylations seem to signal the formation of the caveolin/EGFR complex, a process which is associated with the perinuclear accumulation of EGFR and its persistent kinase activity. A putative nuclear localization sequence (NLS) of EGFR has been identified. Following irradiation EGFR is phosphorylated at residue Thr654 located within this NLS by PKC $\varepsilon$; this phosphorylation induces binding to karyopherin $\alpha$ and karyopherin $\beta$. Overall, the process enables transport through a nuclear pore into the nucleus. Radicals generated by ionizing radiation are also involved in the induction of the transport process [2,5-8]. A model of integral trafficking from the endoplasmic reticulum (ER) to the nuclear envelope (NE) transport (INTERNET) provides another scenario for the nuclear translocation of EGFR, that is, integral inner nuclear membrane (INM) proteins initially inserted into the ER membrane are targeted to the INM of the NE through the outer nuclear membrane (ONM) and nuclear pore complex (NPC). Nuclear EGFR accumulation results from a balance of import and export processes. Recent evidence suggests that nuclear export of EGFR may involve exportin chromosome region maintenance protein 1 (CRM1) $[3,6,9]$.

\section{Role of EGFR in DNA damage repair}

The most important biological effect of ionizing radiation is DNA damage, including single strand breaks (SSBs) and double strands breaks. The most lethal radiation-induced DNA damage is the DSBs. In higher eukaryotes, two distinct mechanisms for DSB repair complement each other to protect cells from radiation-induced DNA damage; these are nonhomologous end-joining (NHEJ) and homologous recombination (HR). NHEJ is operative in all phases of the cell cycle, but especially in G0 and G1 phases, and is the dominant pathway in the repair of DSBs. HR is functional only during the late $\mathrm{S} / \mathrm{G} 2$ phases, when a sister chromatid is available for repair, and it plays a supportive role. These two repair pathways have a close relationship with the phosphoinositide 3-kinase related kinases (PIKKs), including ataxia telangiectasia mutated (ATM), ATM- and Rad3related (ATR) and DNA-dependent protein kinase (DNAPK). EGFR modulates DSB repair primarily via NHEJ, but also via HR [1,10].

\subsection{EGFR and the NHEJ mechanism}

NHEJ is the physical rejoining of DSB ends. NHEJ is catalyzed by a core complex that consists of four proteins, DNA-PK, X-ray repair complementing defective repair in Chinese hamster cells 4 (XRCC4), DNA ligase IV and Artemis, which further associates with a complex of three proteins, Mre11, Rad50, and Nbs1 (MRN), and brings about the physical rejoining of DSB ends [2]. NHEJ is dependent on DNA-PK and so called DNA-PK-dependent nonhomologous end-joinding (D-NHEJ). DNA-PK is a nuclear serine/threonine kinase whose activity is stimulated by double-stranded DNA. DNA-PK comprises three subunits, the $470 \mathrm{kD}$ catalytic subunit (DNA-PKcs), and two regulatory subunits, the $70 \mathrm{kD} \mathrm{Ku} 70$ and the $80 \mathrm{kD} \mathrm{Ku} 80$ [1,2,10]. Phosphorylation of DNA-PKcs at Thr2609 is an essential prerequisite for NHEJ. The effect of EGFR on NHEJ is due to the redistribution and activation of DNA-PK.

(i) The EGFR-DNA-PK complex. DNA-PKcs and $\mathrm{Ku} 70 / \mathrm{Ku} 80$ are considered predominantly nuclear proteins, but there are reports that DNA-PK subunits are independently localized in lipid rafts, where they may interact with membrane proteins also localized in lipid rafts. Ionizing radiation can induce translocation of DNA-PKcs from the nucleus to the cytosol [8]. In the human bronchial carcinoma cell line A549, the human squamous carcinoma cell line $\mathrm{FaDu}$ and the transformed human fibroblast cell line $\mathrm{HH} 4 \mathrm{dd}$, ionizing radiation results in a clear translocation of the perinuclear EGFR to the nucleus along with the nuclear import of Ku subunits 80 and 70 and phosphatase PP1 [5,7]. The EGFR-DNA-PK complex is formed in the nucleus during the first $5 \mathrm{~min}$ after irradiation, concomitant with an increase in activity of DNA-PK, and the maximal activity was detected $10 \mathrm{~min}$ after irradiation [7,11]. Activity of DNA-PK is regulated by phosphorylation and dephosphorylation. Autophosphorylation of DNA-PK at Thr2609, Ser2612, Thr2638 and Thr2647 results in the loss of its protein kinase activity and dissociation of DNA-PKcs from the DNA-bound Ku complex. Protein phosphatases can remove the phosphate groups from autophosphorylated inactive DNA-PKcs and restore DNA-PK protein kinase activity [7].

EGFR makes physical contact with DNA-PK and then forms a complex, which is associated with nuclear translocation of EGFR. As a result of the anti-EGFR antibody C225, which binds to the extracellular domain of EGFR and 
blocks its nuclear translocation, the complex is generated in the cytoplasm. This phenomenon results in a decrease in the nuclear activity of DNA-PK by about $75 \%$, with a concomitant increase in the cytoplasmic activity of DNA-PK; this explains an observed decrease in the efficiency of D-NHEJ $[11,12]$. There are different opinions about whether tyrosine kinase inhibitors that target nuclear kinase activity of EGFR have an effect on the redistribution of DNA-PK. Gefitinib (a tyrosine kinase inhibitor) was observed to suppress nuclear DNA-PK levels in head and neck squamous cell carcinoma cells [13], but not in nonsmall cell lung carcinoma (NSCLC) cell lines A549 [14]. In NSCLC cell lines, unlike wild-type (WT) EGFR, receptors with common oncogenic TKD mutations, L858R or $\triangle \mathrm{E} 746-\mathrm{E} 750$, are more sensitive to ionizing radiation and are defective in radiation induced translocation to the nucleus; receptors with these mutations fail to bind the catalytic and regulatory subunits of the DNA-PK. Cells expressing wtEGFR exhibited greater radiosensitivity when transfected with mtEGFR. DNA-PKcs plays a critical role in EGFR-mediated response to radiation, because in a DNA-PKcs-deficient genetic background, the radioprotective effect of EGFR is lost. Thus, interactions with DNA-PKcs constitute a critical component of EGFRmediated radioprotection $[8,15]$.

(ii) EGFR and phosphorylation of DNA-PK. The full-length EGFR in the nucleus is a tyrosine kinase, shown to be capable of phosphorylating DNA-PKcs at Thr2609 $[2,7,12]$. Although no blockage of nuclear EGFR transport by tyrosine kinase inhibitors was observed in these studies, a clear inhibition of DNA repair was seen. This can be explained by the crucial role of EGFR kinase activity in regulation of DNA-PK or other nuclear proteins involved in DNA repair following irradiation [3].

Epidermal growth factor receptor variant III (EGFRvIII) is the most common type of EGFR mutation; it lacks the ligand-binding domain and is constitutively active. $E G$ FRvIII is the most commonly amplified/mutated gene in glioblastoma multiforme (GBM) and is expressed in about $40 \%$ in head and neck carcinomas. In EGFRvIII-expressing U87 glioma cells, radioresistance is associated with enhanced DSB repair kinetics. Gefitinib and a PI3K inhibitor, LY294002, can abrogate the accelerated DSB repair, while expression of constitutively active, myristylated Akt- 1 accelerates repair. EGFRvIII-expressing U87 glioma cells show elevated activation of DNA-PKcs, and enhanced radioresistance can be abrogated by the DNA-PKcs-specific inhibitor NU7026, but EGFRvIII fails to confer radioresistance in DNA-PKcs-deficient cells. There is no evidence of nuclear translocation of EGFRvIII. It is therefore plausible that EGFRvIII expression in GBM tumor cells might enhance DSB repair via the canonical PI3K/Akt-1 pathway rather than by direct physical association with DNA-PKcs [16]. Activation of DNA-PKcs by IR involves its phosphorylation at several serine/threonine residues.
As Akt-1 is a serine/threonine kinase, it is conceivable that the hyperphosphorylation of DNA-PKcs in EGFRvIIIexpressing cells may be potentiated by activated Akt-1. Knockdown of Akt-1 by specific siRNA blocks DNA-PKcs phosphorylation at Thr2609 and Ser2056 in the human bronchial carcinoma cell line A549 and H460 [16,17]. Blockade of the EGFR-dependent PI3K-Akt pathway in K-RAS-mutated A549 cells significantly affects postradiation survival by diminishing Thr2609 phosphorylation of DNA-PKcs, resulting in a decreased DSB repair capacity. There are strikingly high levels of radiation- induced Akt-1 nuclear translocation in a mouse orthotopic glioma model expressing EGFRvIII. Recently, a close physical association between Akt-1 and DNA-PKcs at sites of DSBs on IR has been shown. DNA-PK-mediated phosphorylation of Akt-1 Ser473 is a key step in activation of Akt-1, and results in a 10-fold enhancement of kinase activity. The cross talk between ATM and DNA-PKcs is probably a central axis in the response of cells to IR-induced DNA damage [1,18-20].

Activation of the RAS/MAPK pathway is an important step in the EGFR-mediated radioprotection, which confers radioresistance by up-regulation of gene transcription involved in DNA repair [21]. Ras activity is responsible for stimulation of PI3K-Akt signaling in response to ionizing radiation, but evidence also indicates an autocrine loop of EGFR stimulation in K-RASmt tumor cells, which activates PI3K-Akt signaling independently of a direct upstream function of K-Ras activity [22]. Constitutively activated, mutant Ras, especially K-Ras, can promote the Ras mutation-dependent up-regulated production and secretion of autocrine EGFR ligands (i.e., TGF- $\alpha$ and amphiregulin); these, in turn, selectively stimulate the EGFR-PI3K-Akt pathway and activated DNA-PKcs.

Phosphorylation of Thr2609 in DNA-PKcs requires ATM, which plays important roles in DNA damage response, cell cycle regulation, and homologous recombination. EGFR can regulate the transcription of the ATM gene [20].

The reviewed experimental data indicate that EGFR directly activates DNA-PK as a tyrosine kinase or indirectly through the activation of the PI3K-Akt pathway or transcriptional regulation of the ATM gene.

There are also data suggesting that EGFR might interact with NHEJ via H2AX or XRCC1. Phosphorylation of $\mathrm{H} 2 \mathrm{AX}$ is considered a signal for the accumulation of repair proteins near damaged chromatin. Consequently, blockage of the EGFR-PI3K-Akt pathway results in inhibition of phosphorylation of $\mathrm{H} 2 \mathrm{AX}$ and thus retards this accumulation impeding DNA repair [23]. XRCC1 is a cofactor of DNA-PKcs and plays a central role in base excision of single-strand break repair. It has been reported that an activation of EGFR by X-irradiation resulted in a rapid upregulation of XRCC1 expression, which was mediated via MAPK signaling [10]. 


\subsection{EGFR and the HR mechanism}

Using the sister chromatid or DNA repeats as templates, HR repairs DSBs. HR is initiated when the DNA single strands emerge after DNA strands are cut by an MRN complex. MRN forms complexes with replication protein $\mathrm{A}$ and Rad51; both are core proteins in the HR machinery. Several studies have reported that MAPK signaling regulates HR in human cells either positively or negatively. When ERK1/2 signaling was stimulated by oncogenic Raf-1, an approximate 2-fold increase in HR was observed. By contrast, inhibition of the p38 MAPK pathway also generated an almost 2-fold stimulation of HR. EGFR and growth factor signaling in general has long been known to modulate cell cycle regulation. It is well-accepted that $\mathrm{HR}$ is most active in late $\mathrm{S}$-phase and G2. It is plausible, therefore, that these apparently disparate changes in HR can be reconciled by considering the cell cycle phase in which they have occurred [18,24,25].

ATM protein plays a crucial role in HR. Upon DNA damage or alterations in chromatin structure, ATM dimers are quickly autophosphorylated at Ser1981, thereby activating ATM and leading to the phosphorylation of several downstream targets, including H2AX, p53, CHK2, Nbs1, hMDM2, SMC1, Rad51 and BRCA1. RAS/RAF/MEK/ ERK signaling is associated with phosphorylation of ATM at Ser1981. Inhibition of MAP/ERK kinase (MEK)/ERK signaling suppresses ATM phosphorylation levels $>85 \%$ throughout the cell cycle. The ATM-specific inhibitor KU-55933 partly blocks radiation-induced ERK1/2 phosphorylation, suggesting that ATM regulates ERK1/2 signaling. Thus, it seems that ATM and ERK signaling might be interacting in a regulatory feedback loop [25,26]. EGFR may affect transcription of the $A T M$ gene by NF- $\mathrm{BB}$, activated through the PI3K-Akt pathway. When NF- $\mathrm{BB}$ is activated it translocates to the nucleus, where it activates ATM, ATR and DNA-PK, thus promoting DNA damage repair and cell proliferation and/or inhibiting apoptosis.

In gefitinib-pretreated A549 cells, a DNA repair protein, pNbs1, exhibits a more robust and prolonged activation after irradiation. Because Nbs1 functions in a protein complex MRN, an abnormal abundance of Nbs1 may sequester other repair proteins away from the sites of DSBs. Nbs1 is a substrate for the ATM kinase; Nbs1 phosphorylation is a possible connection between EGFR $>$ ERK $>$ ATM $>$ Nbs 1 , and should be examined in further studies [14].

In human breast cancer cells, blockage of EGFR by erlotinib attenuates DNA damage-induced Rad51 foci and results in a substantial reduction of BRCA1 available in the nucleus to perform HR. Both Rad51 and BRCA1 are essential components of the HR machinery [27]. Treatment of NSCLC cells with gefitinib resulted in decreased levels of phospho-ERK1/2 and Rad51 protein and their mRNAs. The potent MEK inhibitor, U0126, mimicked the effects of gefitinib suggesting that MEK/ERK signaling downstream of
EGFR regulates HR by controlling the levels of Rad51 [24]. EGFR may affect phosphorylation of BRCA1 via the PI3K/ Akt pathway, because Akt-1 can directly phosphorylate BRCA1 on Thr509 [1].

EGFR may interact with proliferating cell nuclear antigen (PCNA) as well as with components involved in NHEJ and HR. PCNA is subject to tyrosine phosphorylation at Tyr211 in an EGFR-dependent manner, and this phosphorylation enhances PCNA stability on chromatin. PCNA is an essential protein for DNA synthesis, damage repair and cell proliferation. Isoforms of PCNA, including Rad9, Hus1 and Rad1, assemble in a homotrimeric ring structure encircling the DNA double helix; in this format it functions as a sensor for DNA damage and is involved in DNA replication. Phospho-PCNA is correlated with nuclear EGFR in human breast cancer tumor samples and has a strong prognostic value for breast cancer patients survival. Nuclear EGFR is also positively correlated with the expression of PCNA in oral squamous cancer [28].

In summary, EGFR promotes DSB repair by interacting with proteins that implement NHEJ and HR, including DNA-PK, ATM, Rad51 and BRCA1 (in every case as the nuclear translocated version), via the PI3K-Akt and Ras-Raf-MAPK pathways (Figures 1 and 2).

\section{Future prospects}

DNA repair is emerging as an attractive pharmacological target in chemotherapy and radiotherapy. Although many solid tumors may escape the process of apoptosis, the

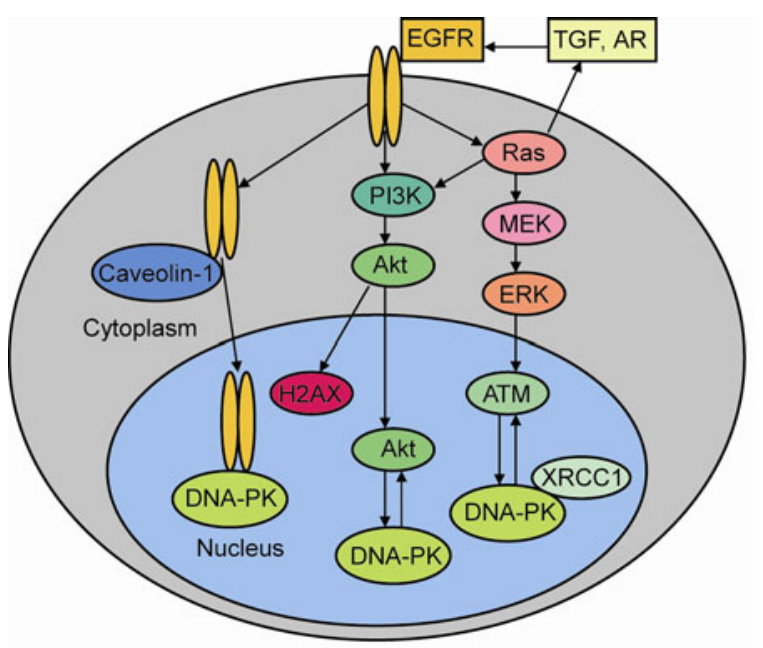

Figure 1 Mechanisms that have been identified to explain how EGFR signaling interacts with NHEJ. Upon ionizing irradiation, EGFR modulates the activity of DNA-PK, a key component in NHEJ, by various means: (1) EGFR translocates to the nucleus and forms a complex with DNA-PK; (2) EGFR activates DNA-PK directly as a tyrosine kinase; (3) EGFR activates DNA-PK through the activation of the PI3K/Akt pathway; (4) EGFR regulates the transcription of $A T M$ and $X R C C 1$ genes, which are associated with DNA-PK via the ERK pathway. 


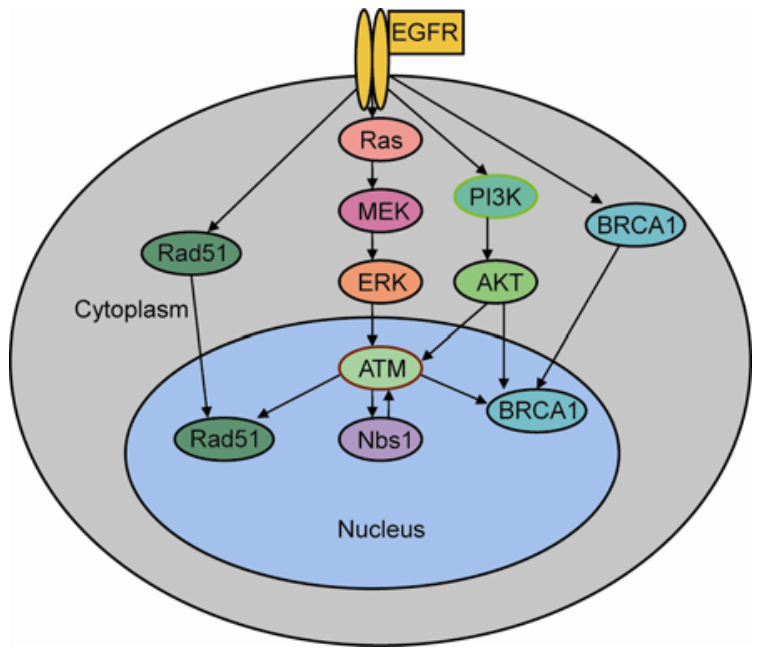

Figure 2 Mechanisms that have been identified to explain how EGFR signaling interacts with HR. EGFR regulates the activity and transcription of $A T M, B R C A 1$ and Rad51 mainly through the RAS/RAF/MEK/ERK pathway and PI3K/Akt pathways.

mechanisms underlying DNA damage repair remain fundamentally the same as those in normal cells. Inhibitors that target DNA-PK (NU7441) and ataxia telangiectasiamutated (KU55933), have shown promising results in augmenting the effects of radiation in preclinical studies and clinical trials. Disruption of EGFR nuclear import also compromises DNA repair and tumor survival. A therapeutic strategy that disrupts EGFR nuclear import or abrogates EGFR-DNA-PKcs interactions could potentially be tumor-specific, sparing normal tissue. EGFR expression negatively correlates with the outcomes of radiotherapy in EGFR-overexpressed head and neck carcinomas. A greater molecular understanding of the novel connections between EGFR signaling and DSB repair should fuel the development of even more refined radiosensitizing approaches in the future.

The authors gratefully acknowledge the comments and suggestions of Dr. Robert Wohlhueter. This work was supported by the National High Technology Research and Development Program of China (2009AA02Z403) and the National Natural Science Foundation of China (30873010 and 81072220).

1 Mukherjee B, Choy H, Nirodi C. Targeting nonhomologous end-joining through epidermal growth factor receptor inhibition: Rationale and strategies for radiosensitization. Semin Radiat Oncol, 2010, 20: 250-257

2 David J C, Chaitanya S N. The epidermal growth factor receptor: A role in repair of radiation-induced DNA damage. Clin Cancer Res, 2007, 13: 6555-6560

3 Dittmann K, Mayer C, Rodemann1 H P. Nuclear EGFR as novel therapeutic target. Insights into nuclear translocation and function. Strahlenther Onkol, 2010, 186: 1-6

4 Tao Y G, Song X, Deng X Y, et al. Nuclear accumulation of epidermal growth factor receptor and acceleration of G1/S stage by Epstein-Barr-encoded oncoprotein latent membrane protein 1. Exp Cell Res, 2005, 303: 240-251
5 Szumiel I. Epidermal growth factor receptor and DNA double strand break repair: The cell's self-defence. Cell Signal, 2006, 18: 15371548

6 Dittmann K, Mayer C, Kehlbach R, et al. Radiation-induced caveolin-1 associated EGFR internalization is linked with nuclear EGFR transport and activation of DNA-PK. Mol Cancer, 2008, 7: 69-78

7 Dittmann K, Mayer C, Fehrenbacher B, et al. Radiation-induced epidermal growth factor receptor nuclear import is linked to activation of DNA-dependent protein kinase. J Biol Chem, 2005, 280: 31182-31189

8 Liccardi G, Hartley J A, Hochhauser D. EGFR nuclear translocation modulates DNA repair following cisplatin and ionizing radiation treatment. Cancer Res, 2011, 71: 1-12

9 Wang Y N, Yamaguchi H, Hsu J M. Nuclear trafficking of the epidermal growth factor receptor family membrane proteins. Oncogene, 2010, 29: 3997-4006

10 Kriegs M, Kasten-Pisula U, Rieckmann T. The epidermal growth factor receptor modulates DNA double-strand break repair by regulating non-homologous end-joining. DNA Repair, 2010, 9: 889-897

11 Bandyopadhyay D, Mandal M, Adam L, et al. Physical interaction between epidermal growth factor receptor and DNA-dependent protein kinase in mammalian cells. J Biol Chem, 1998, 273: 1568-1573

12 Hsu S C, Miller S A, Wang Y. Nuclear EGFR is required for cisplatin resistance and DNA repair. Am J Transl Res, 2009, 1: 249-258

13 Shintani S, Li C, Mihara M, et al. Enhancement of tumor radioresponse by combined treatment with gefitinib (Iressa, ZD1839), an epidermal growth factor receptor tyrosine kinase inhibitor, is accompanied by inhibition of DNA damage repair and cell growth in oral cancer. Int J Cancer, 2003, 107: 1030-1037

14 Tanaka T, Munshi A, Brooks C, et al. Gefitinib radiosensitizes non-small cell lung cancer cells by suppressing cellular DNA repair capacity. Clin Cancer Res, 2008, 14: 1266-1273

15 Das A K, Chen B P, Story M D, et al. Somatic mutations in the tyrosine kinase domain of epidermal growth factor receptor (EGFR) abrogate EGFR-mediated radioprotection in non-small cell lung carcinoma. Cancer Res, 2007, 67: 5267-5274

16 Mukherjee B, McEllin B, Camacho C V. EGFRvIII and DNA double-strand break repair: A molecular mechanism for radioresistance in glioblastoma. Cancer Res, 2009, 69: 4252-4259

17 Toulany M, Kehlbach R, Florczak U, et al. Targeting of AKT1 enhances radiation toxicity of human tumor cells by inhibiting DNAPKcs-dependent DNA double-strand break repair. Mol Cancer Ther, 2008, 7: 1772-1781

18 Golding S E, Morgan R N, Adams B R. Pro-survival AKT and ERK signaling from EGFR and mutant EGFRvIII enhances DNA double-strand break repair in human glioma cells. Cancer Biol Ther, 2009, 8: 730-738

19 Bozulic L, Surucu B, Hynx D, et al. PKBa/Akt1 acts downstream of DNA-PK in the DNA double strand break response and promotes survival. Mol Cell, 2008, 30: 203-213

20 Chen B P, Uematsu N, Kobayashi J, et al. Ataxia telangiectasia mutated (ATM) is essential for DNA-PKcs phosphorylations at the Thr-2609 cluster upon DNA double strand break. J Biol Chem, 2007, 282: 6582-6587

21 Weidhaas J B, Eisenmann D M, Justin M, et al. A conserved RAS/mitogen-activated protein kinase pathway regulates DNA damage-induced cell death postirradiation in radelegans. Cancer Res, 2006, 66: 10434-10438

22 Toulany M, Baumann M, Rodemann H P. Stimulated PI3K-Akt signaling mediated through ligand or radiation-induced EGFR depends indirectly, but not directly, on constitutive K-ras activity. Mol Cancer Res, 2007, 5: 863-872

23 Toulany M, Kasten-Pisula U, Brammer I. Blockage of epidermal growth factor receptor-phosphatidylinositol 3-kinase-AKT signaling increases radiosensitivity of K-RAS mutated human tumor cells in vitro by affecting DNA repair. Clin Cancer Res, 2006, 12: 41194126

24 Meyn R E, Munshi A, Haymach J V. Receptor signaling as a regulatory mechanism of DNA repair. Radiother Oncol, 2009, 92: 316-322 
25 Golding S E, Rosenberg E, Neill S, et al. Extracellular signal-related kinase positively regulates ataxia telangiectasia mutated, homologous recombination repair, and the DNA damage response. Cancer Res, 2007, 67: 1046-1053

26 Nyati M K, Feng F Y, Maheshwari D, et al. Ataxia telangiectasia mutated down-regulates phospho-extracellular signal-regulated kinase $1 / 2$ via activation of MKP-1in response to radiation. Cancer Res,
2006, 66: 11554-11559

27 Li L P, Wang H, Eddy S, et al. Erlotinib attenuates homologous recombinational repair of chromosomal breaks in human breast cancer cells. Cancer Res, 2008, 68: 9141-9146

28 Wang S C, Nakajima Y, Yu Y L, et al. Tyrosine phosphorylation controls PCNA function through protein stability. Nat Cell Biol, 2006, 8: $1359-1368$

Open Access This article is distributed under the terms of the Creative Commons Attribution License which permits any use, distribution, and reproduction in any medium, provided the original author(s) and source are credited. 